\title{
IPTV Quality assessment system
}

\author{
Luis Casadesus, Julián Fernández-Navajas, Luis Sequeira, Idelkys Quintana, Jose Saldana, \\ José Ruiz-Mas \\ Communications Technology Group (GTC)-Aragón Inst. of Engineering Research (I3A) \\ Dpt. IEC. Ada Byron Building. EINA. Univ. Zaragoza \\ 50018 Zaragoza, Spain \\ Email: \{luis.casadesus, navajas, sequeira, idelkysq, jsaldana, jruiz\}@unizar.es
}

\begin{abstract}
Due to the Increasing deployment of real-time multimedia services like IPTV and videoconferencing, the Internet has new Challenges. These new real-time Applications require a reliable performance of the network so as to provide a good Quality of Service (QoS) so it is important for the services providers to estimate the quality offered; and regardless of the transport network to know the quality perceived by the user. For this it is important to have tools to evaluate the quality of service provided. This paper presents a system for IPTV quality assessment. This will allow us to study the user's perceived quality for different codecs, bit rates, frame rates and video resolutions, and the impact of the network packet loss rate, in order to determine the objective and subjective quality. We propose an application simulating packet loss as a function of network parameters, which can be used to obtain the received video with different network impairments, without the need for transmitting it. It has two main advantages: first, it avoids the need of transmitting the video a number of times; second, it allows test repeatability.
\end{abstract}

\section{General Terms}

Measurement, Performance, Experimentation.

\section{Keywords}

Quality assessment, quality of service (QoS), quality of experience (QoE), video streaming, IPTV, metrics

\section{INTRODUCTION}

The rapid growth of the Internet and the increase of computing power have caused the raising of a number of applications. Among them, real-time multimedia services, as Voice over IP (VoIP), video conferencing, IP Television (IPTV) or telemedicine, are getting more and more popular. The multimedia contents that are experiencing the biggest boom are related to video [1].

These new real-time applications require a reliable performance of the network so as to provide a good Quality of Service (QoS) [2], defined in [3] as "the totality of characteristics of a telecommunications service that bear on its ability to satisfy

Permission to make digital or hard copies of all or part of this work for personal or classroom use is granted without fee provided that copies are not made or distributed for profit or commercial advantage and that copies bear this notice and the full citation on the first page. To copy otherwise, or republish, to post on servers or to redistribute to lists, requires prior specific permission and/or a fee.

LANC'12, October 4-5, 2012, Medellin, Colombia.

Copyright $(92012$ ACM 978-1-4503-1750-4/12/10 ...\$15.00. stated and implied needs of the user of the service."

The perception that end users have on how they are being provided a given service is the way to describe the concept of Quality of Experience (QoE). P.10/G.100 ITU-T defines it as "the global acceptance of an application or service as perceived by the end user, where such acceptance may be influenced by the user's own expectations or the context in which it develops." Work in progress of ITU-T presents the QoE as the combination of objective components, expressed as QoS, and subjective components, expressed as human factors. Traditionally, the QoE has been quantified with subjective methods such as the MOS (Mean Opinion Score) assuming a correlation between QoS parameters and MOS. Examples of this are the recommendations ITU-T P.800, P.910, P.920 and P.930 that gather the most relevant aspects for analysis of quality in broadband IP services. However, in order to make the quantification of QoE more complete and to allow the inclusion of a real environment, the transformations performed should be extended to the different flows in the existing IP broadband services. The underlying network technology [4] [5] and the most relevant QoS parameters [6] have to be taken into account for a complete quantification.

As said in [1], the video is a key element in the development of the Internet in the next few years, so many researchers have focused on developing better quality metrics. Determining the quality of a video is a complex problem, because of the characteristics of the video transmission process may impair its quality: content type, bit rate, frame rate, resolution, codec, QoS parameters, human perception (expectation, ambiance).

The main network parameters normally considered to determine the QoS for real-time multimedia applications are the capacity, available bandwidth, delay, delay variation and packet loss rate. The problem arises when attempting to relate these parameters with the quality perceived by the user of a service (QoE).

In order to achieve a quality assessment of multimedia services, it is important to know the schemes to determine the quality and the metrics to use, the employed compression methods and how they work, the effects that may modify user's perception and how all these things are related. To find this relationships it is important to have tools that allow to perform tests to assess the quality in a manner as close as possible to the perception of the final user.

In this paper we present a system for IPTV quality assessment. This will allow us to study the user's perceived quality for different codecs, bit rates, frame rates and video resolutions, and the impact of the network packet loss rate, in order to determine the objective and subjective quality. We propose an application simulating packet loss as a function of network parameters, which can be used to obtain the received video with different network impairments, without the need for transmitting it. It has two main 
advantages: first, it avoids the need of transmitting the video a number of times; second, it allows test repeatability.

This paper is organized as follows: Section II discusses an overview of different video quality assessment techniques. Section III covers the architecture and modules of the proposed system. Section IV describes a brief test for validating the implementation and Section IV concludes the paper.

\section{OVERVIEW OF QUALITY ASSESSMENT}

When we design a system for assessing the quality of multimedia streaming arise many questions, where do the measurements?, how to do it? when? what information is going to be used? what modifies de user perception?. This section shows a summary of the issues involved in video streaming system to answer these questions. A general IPTV transmission scheme is shown in figure 1.

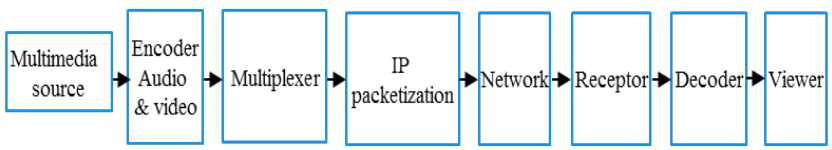

Figure 1. IPTV transmission scheme.

In general, measurements of QoS parameters can be done in three ways: Full reference (FR) model: Original and received content are available for evaluation. No reference (NR) model: Quality information has to be extracted from the received content as no reference is available. Reduced reference (RR) model: The same parameters are derived and compared, usually using an alternative channel to transmit the parameters between sender and receiver.

In [7] a number of objective quality assessment models are classified into five categories, as a function of the type of information they handle: media-layer model use the media signal to obtain the metric value; the parametric packet-layer model utilizes the packet-header (such as RTP headers) information to predict QoE without handling the media signal itself; the parametric planning model uses quality planning parameters for networks, thus needing information a priori about the system under test; the bitstream layer model utilizes information from the encoded bitstream and from the packet header, mixing the two previous models; finally, the hybrid layer model is a combination of the previously mentioned ones, exploiting as much information as possible.

Video quality measurement techniques include pre-service and inservice tests. The pre-service tests are performed offline. They are mainly used for full-reference measurement technique, and developed in laboratory environments. The in-service tests are performed online. They are mostly used with free-reference measurement techniques because they require real-time capabilities, i.e. identifying impairments as they happen during data transmission.

\subsection{Video quality assessment metrics}

There are several video quality assessment metrics proposed [8] [9] using different types of data at different layers. One of the most relevant objective metrics in the area of video quality assessment is Peak Signal to Noise Ratio (PSNR) [10], a measurement that compares pixel by pixel and frame by frame the original and received video signals. It is a good approximation, although it ignores the spatial and temporal relationships of the pixels, thus resulting on video distortions that have similar PSNR values but different quality perception. It is based on FR and media-layer models.

To address this shortcoming, there are two metrics that aim to analyze and extract certain features of the video. Structural Similarity (SSIM) [11] combines luminance, contrast and structural similarity of the image to compare the correlation and Video Quality Metric (VQM), considering blurring, global noise, and block and color distortions [12].

The Moving Picture Quality Metric (MPQM) is an objective quality metric for moving pictures using vision modeling approach [13], incorporating two human vision characteristics: contrast sensitivity and masking. Color MPQM is an extension of the MPQM metric which also considers the effect of chrominance on the quality. V-Factor is a particular implementation of MPQM for IPTV, which attempts to rate the perceived quality from 1 to 5 .

The MOS is the most widely used subjective metric. Many parameters of the viewing conditions can influence the results, such as room illumination, type of display, brightness, contrast, resolution, viewing distance, and the age and educational level of experts. The methods used to perform rating experiments and obtain the MOS are described in ITU-R BT.500-13 [14]. It describes how sequences are presented to experts and how their opinion is collected. It recommends the double-stimulus impairment scale (DSIS) method by which the individuals punctuate degradation test sequence with respect to a reference and the double-stimulus continuous quality-scale (DSCQS) by which the individuals compare pairs of short sequences: one using the process under examination and its corresponding reference.

\subsection{MPEG compression.}

The Motion Picture Expert Group (MPEG) has produced several standards for video compression. MPEG uses three basic techniques for compression: firstly, subsampling, reducing the number of colors that are less sensitive to the human eye. Secondly, spatial compression or intra coding, remove redundant information within frames, using the property that the pixels within a frame are related to their neighbors. And thirdly, temporal compression or inter-frame coding, removing redundant information between subsequent frames.

An MPEG encoder converts and compresses a video signal into a series of pictures or frames; basically there are three types of frames: the I-frames (Intra frames) contain a complete picture and are coded without reference to others. The P-frames (Predictive coded frames) are encoded using the preceding I or P-frame using temporal compression. The B-frames (Bi-predictive coded frames directional) use the previous and next $\mathrm{I}$ or P-frame as their reference points for motion compensation. They are the ones which require less bytes.

Frames are grouped into Group of Pictures (GoP). A GoP contains an I-frame and all frames associated with it. An MPEG stream is formed by consecutive GoPs, each of them beginning with an Iframe followed by $\mathrm{P}$ and B-frames. Each frame is formed by sections; each section is composed of various macroblocks. These macroblocks are a group of blocks, which encode the pixels shown in the image.

In MPEG-4 AVC/H.264, B-frames can or cannot be used as a reference, and there are two more frame types, namely SP (Switching P) and SI (Switching I), used to encode the transition between two video streams. 
MPEG-2 and MPEG-4 are the most widely used encoding schemes for television applications today. ITU Rec. G.1080 $(12 / 08)$ recommends for Standard Definition (SD) broadcast the codec MPEG-2-part-2(h.262) Main profile at Main level using $2.5 \mathrm{Mbit} / \mathrm{s}$ at constant bitrate (CBR) and MPEG-4 AVC (Main profile at Level 3.0) at $1.75 \mathrm{Mbit} / \mathrm{s}$ CBR. For High Definition (HD) broadcast, it recommends h.262 at Main profile at Main level, using $15 \mathrm{Mbit} / \mathrm{s}$ CBR and MPEG-4 AVC (Main profile at Level 4) at $10 \mathrm{Mbit} / \mathrm{s}$ CBR [19].

\subsection{MPEG IP Encapsulation}

To transport MPEG-encoded video over IP networks, MPEG frame information is encapsulated within MPEG Transport Stream (TS) packets. Transport Stream is specified in MPEG-2 Part 1 and it is used in broadcast systems such as DVB, ATSC and IPTV. An IP packet for transporting MPEG video usually contains seven 188-byte MPEG-TS packets. This fact is important, since a single frame (I, P, B) can be split into different IP packets for transmission; in addition, an IP packet may carry audio and video data. Figure 2 shows an IP packet encapsulating TS packets

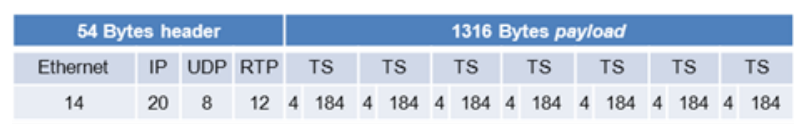

Figure 2. TS packet encapsulated for transmission.

\subsection{What modifies the user perception.}

Regardless viewing conditions and the human factor, what modifies the user perception? The concept of video artifact can be useful in order to answer this question: it is a visible distortion on a displayed video image relative to the original image [15]. These are the main effects perceived by the user when watching a video:

Video blockiness is an impairment in which the image contains artifacts that resemble small blocks of a single color. It is caused by block-based coding schemes. It is seen by the viewer as one large pixel being displayed over a large area of the screen.

Video blurriness has the same root cause as blockiness, i.e. insufficient availability of bits to encode all the details of the image. Advanced video coding algorithms that enable the encoder to use bits more efficiently can decrease the effect of video blurriness.

Video freezing: the video is interrupted while it is being watched. This is typically caused by insufficient bandwidth, buffering problems or delayed or lost packets.

Video jerkiness: the video stream might not flow smoothly, and the action might appear to start and stop rapidly. This is often caused by insufficient bandwidth, buffering problems or delayed or lost packets.

Video blackout: the video content completely disappears, this is caused by a complete loss of the transmitted signal, although it is more often caused by severe bandwidth issues.

For user's perception it is also important the perfect synchronization between the audio and video streams. Some audio sync problems can be attributed to bandwidth issues, or also to a bad alignment at the time of content creation. This was studied in [16], using four videoconferencing applications.

Other metrics related to the delivered service are also important for the user's perception. First, user synchronization affects applications performing live streaming in P2P networks [17]. As an example, a person watching a soccer game does not want to hear the goal scream of the neighbors before viewing it on the TV. The start-up time, i.e. the delay since the user queries a program and receives the content, is also important. This is also related to zapping time, which is a key element in IPTV: how quickly the users can change between channels. In [18] various experiments related to zapping time were conducted.

\subsection{Standards}

Research in this area has resulted in numerous standards, some of them already mentioned above. These are the ones employed for objective measurements:

ITU-T J.148 (05/2003) Requirements for an objective perceptual multimedia quality model.

ITU-T J.144 (03/2004) Objective perceptual video quality measurement techniques for digital cable television in the presence of a full reference.

ITU-T G.1070 (04/2007): Opinion model for video-telephony applications.

ITU-T G.1070 Amd. 1 (11/2009) - Coefficients in video quality estimation function with respect to coding and packet-loss degradations.

ITU-T J.246 (08/2008) Perceptual visual quality measurement techniques for multimedia services over digital cable television networks in the presence of a reduced bandwidth.

ITU-T J.247 (08/2008) Objective perceptual multimedia video quality measurement in the presence of a full reference.

ITU-T J.249 (01/2010) Perceptual video quality measurement techniques for digital cable television in the presence of a reduced reference.

ITU-T J.342 (04/2011) Objective multimedia video quality measurement of HDTV for digital cable television in the presence of a reduced reference signal

For subjective measurements:

ITU-T P.800 (08/1996) Methods for subjective determination of transmission quality

ITU-R Rec BT.500-13 Methodology for the subjective assessment of the quality of television pictures

ITU-T P.910 (04/2008) Subjective video quality assessment methods for multimedia applications

ITU-T P.911 (12/1998) Subjective audiovisual quality assessment methods for multimedia applications

Part of the emergence of these standards is due to the work of the Video Quality Experts Group (VQEG). It was born from a need to bring together experts in subjective video quality assessment and objective quality measurement. VQEG has been acting as a technical advisory team.

For audio and video synchronization:

ITU-R BT.1359-1 (01/1998): Relative Timing of Sound and Vision for Broadcasting.

We can note that more research is needed to achieve standards in QoE on multimedia services.

\subsection{Tools}

There are several tools related to the issues we have seen, one of the tools most cited on the Internet is Evalvid [20]; it is a set of 
open source tools for the evaluation of the quality of video transmitted through a communications network. Offers values of delay, jitter and loss, and performs measurements of video quality (PSNR, SSIM, MOS). It is developed in C, and although it is good framework does not facilitate the extension of the tools to add further support to codecs, metrics or different models of losses for simulation. Evalvid has problems when there are losses and frames are out of alignment, the PSNR value lost reliability. In [21], they added new agents to achieve better reliability, and enable Evalvid to link seamlessly with NS2.

Evalvid-RA [22] it is an add to Evalvid for the simulation of rate adaptive video. The solution generates real rate adaptive MPEG-4 streaming traffic, using the quantizer scale for adjusting the sending rate.

Other tool recently added to VQEG group it is Sirannon [23] [24], a modular media streamer and receiver offering a configuration method based on graphs of components. It supports multiple codecs and multiple transport mechanism. Although it does not compute metrics it is a great tool to experiment and research in these topics.

\section{SYSTEM ARCHITECTURE.}

After presenting a theoretical study of the major issues affecting the video streaming quality, we present the architecture of the system we are proposing. The tool allows calculate FR objective metrics like PSNR and SSIM using MPEG compression. MPEG is encapsulated in TS packets. It is also possible to obtain MOS subjective metric in a NR scheme. Hybrid layer model is in general used. The tool was developed in $\mathrm{C}++$ language.

Our goal is to obtain a set of received IPTV video broadcasts, when the original signal has been transmitted through a network with variable parameters. For doing this, a first method could be transmitting this video a number of times, using the suitable network parameters for each run. Instead of doing this, an application simulating packet loss as a function of network parameters is proposed, which can be used to obtain the received video with different network impairments, without the need for transmitting it. It has two main advantages: first, it avoids the need of transmitting the video a number of times; second, it allows test repeatability.

The application simulates possible errors occurring during the transmission of the encoded video through an IP network, using different packet loss models and different packetization schemes, by varying the IP MTU (Maximum Transfer Unit) (Figure 3). The application can be used with different codecs and packet loss models and it is easily extensible.

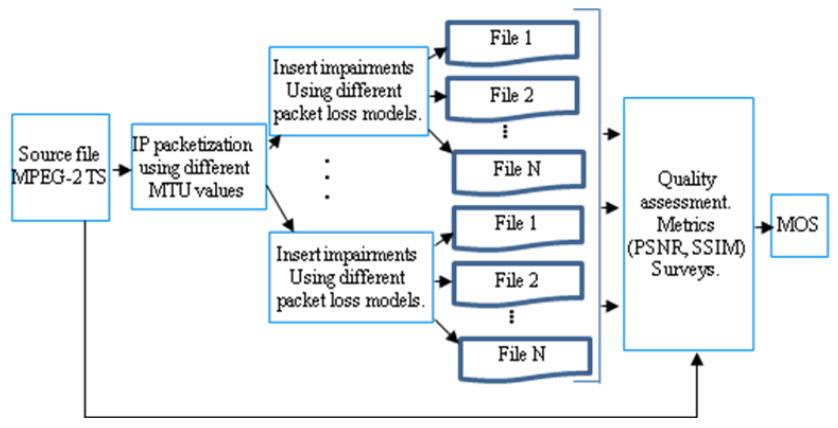

Figure 3. Application scheme

In Figure 4 we can see how to add a new video or audio codec and in Figure 5 how to add a new model of losses.

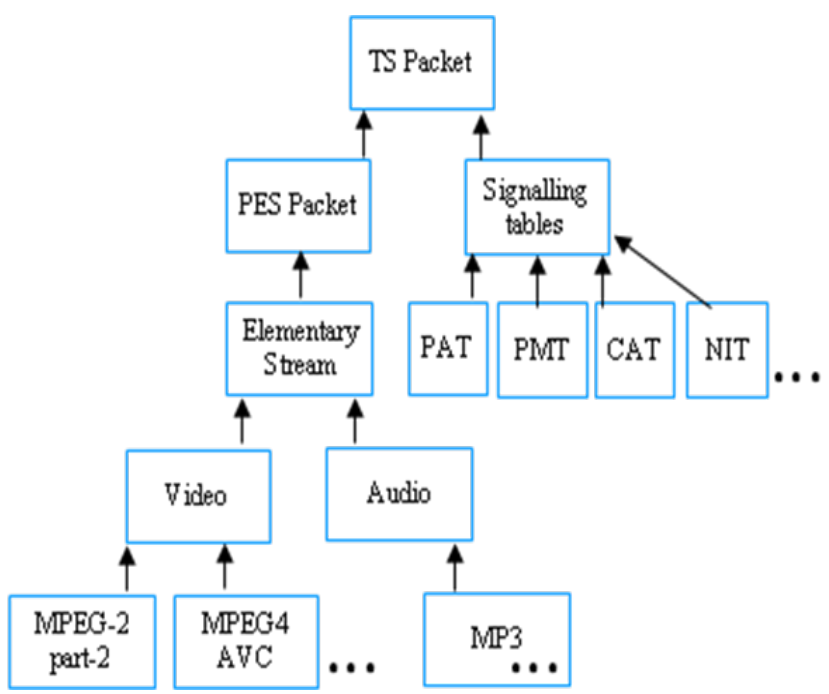

Figure 4. Diagram of application classes.

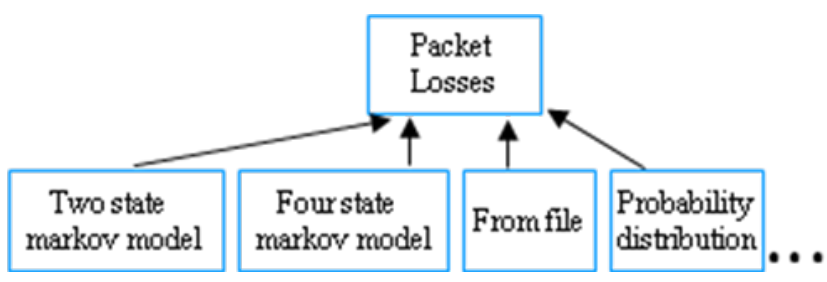

Figure 5. Diagram of application classes.

The system has four functional modules: IP Packetizer, Packet loser, Loss inspector and Quality analyzer. The Figure 6 shows the interaction of the application modules. The encoding and decoding are made using FFmpeg [25] and x264 [26] but can be used any encoder. The Loss inspector implements h.262 and h. 264 codecs. The other modules are codec independent.

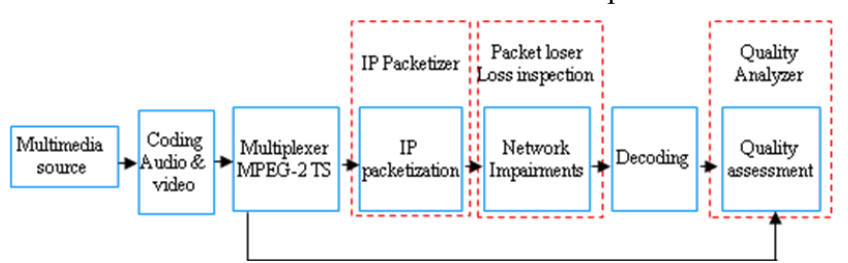

Figure 6. Functional modules.

\subsection{IP Packetizer}

The IP packetizer is responsible for generating the network packet traces (time and packet size). It receives as parameters the MTU and a time interval in order to smooth the sending of packets to the network, ensuring a constant bitrate between the two extremes and thus avoiding bitrate peaks when a frame is large (e.g. frames being used as reference). Based in the multimedia type the TS streaming can be split in audio and video packets (Figure 7). 


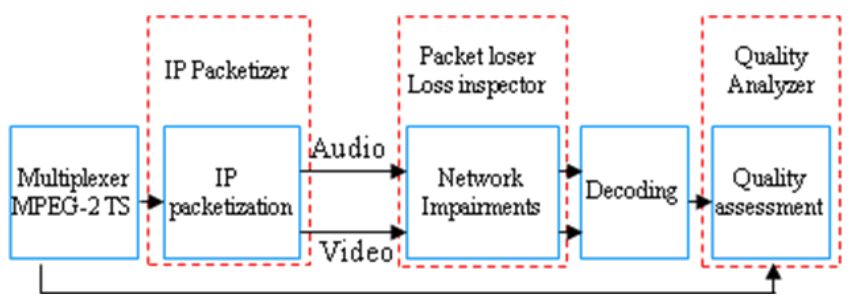

Figure 7. Audio and video packetization for differents channels.

\subsection{Packet loser}

Packet loss can be simulated in various ways. Taking into account that packet losses occur in the Internet in a bursty manner, due to buffer overflows or router updates or disconnections, a common model used to characterize the behavior of a bursty channel is the Gilbert-Elliott 2-state Markov approach [27] [28], based on a discrete two-state model with at first a good (G) and secondly a bad (B) state with different loss rates and transition probabilities from states. It can also be used a 4-state Markov model [29] with two good and bad states to generate a hypergeometrical distribution of the duration of good and bad phases [30]. Both the bad and the good states represent the dependency between consecutively lost or found packets. The models can be used with time of lost instead of probability of lost in the states; for example you can simulate the loss of $100 \mathrm{~ms}$ of packets (Figure 8).

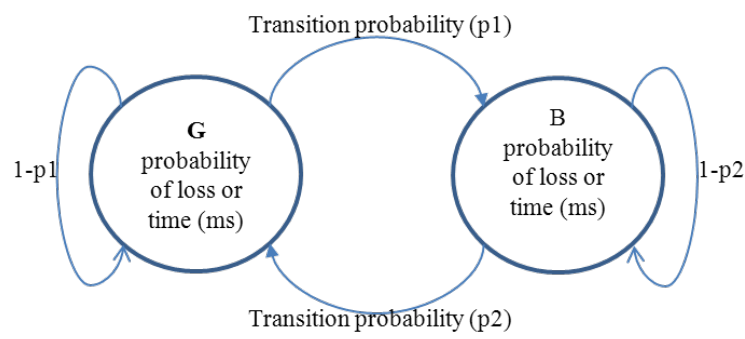

Figure 8. Two state markov chain.

Another possibility is using a file with real packet loss values taken from measurements of actual networks. Finally, we can use a probabilistic distribution function.

\subsection{Loss inspector}

Responsible for inspecting packets to see what removes the packet loser. It receive the complete frame. Generates a trace file with which part and frame type (I,P,B) has been lost. Implements h.262 and h.264 codecs.

\subsection{Quality Analyzer}

This module is responsible for performing quality measurements. Using as an input the result files, it can calculate PSNR and SSIM, applying a FR model using original compressed video and video with impairments. We can also conduct surveys to obtain the end user's experienced video quality.

\section{Test and results.}

In this section we explain the test which has been conducted for validating the application. We used the movie "Elephant dreams" [31], 1minute, from minute 1 to minute 2. Each experiment has been made 20 times in order to obtain the results shown in Table I. Packet loss has been simulated using a two state Markov model, with different losses. Different values for MTU were used (500, 1000 and 1500).

The video was encoded as:

codec: h264 mpeg-4 AVC (part 10)

resolution: $1920 \times 1080$

frame rate: 24

bitrate: $10 \mathrm{Mbps}$

GOP size: 24

B-frames: 2

h.264 level: 4

h.264 profile: high

Table I. Values of PSNR and SSIM for different MTU and losses.

\begin{tabular}{|l|l|l|l|l|l|}
\hline MTU & $\begin{array}{l}\text { Losses } \\
(\%)\end{array}$ & PSNR & $\begin{array}{l}\text { PSNR } \\
\text { SD. }\end{array}$ & SSIM & $\begin{array}{l}\text { SSIM } \\
\text { SD. }\end{array}$ \\
\hline 500 & 1 & 26,16 & 5,93 & 0,87 & 0,15 \\
\hline & 2 & 23,04 & 4,43 & 0,78 & 0,16 \\
\hline & 3 & 21,67 & 4,15 & 0,75 & 0,17 \\
\hline 1000 & 1 & 26,46 & 7,58 & 0,86 & 0,15 \\
\hline & 2 & 23,04 & 5,57 & 0,79 & 0,18 \\
\hline & 3 & 21,40 & 5,36 & 0,76 & 0,19 \\
\hline 1500 & 1 & 26,47 & 9,10 & 0,85 & 0,16 \\
\hline & 2 & 24,33 & 7,11 & 0,80 & 0,18 \\
\hline & 3 & 21,87 & 5,46 & 0,75 & 0.2 \\
\hline
\end{tabular}

We can see in Table I that if the losses increase then logically the PSNR and SSIM values decrease. It can be observed also that the packets size does not affect the values in this case. These results may be different in a real network due to the different buffers implementations. Therefore more research with different videos and scenarios are needed.

\section{Conclusions and future work}

In this paper we introduce an overview of existing techniques for video quality assessment. We reviewed the models that can be used depending of the information available, the most suitable metrics, the main codecs used, the way to encapsulate the audio and video over the network for IPTV service and what perceive the user that change his perception.

Then we present the architecture and workflow of an application for IPTV quality assessment. The application has four functional modules: IP Packetizer, Packet loser, Loss inspector and Quality analyzer. They allow the simulation of packet loss in a TS streaming and obtain videos with different impairments without transmit it on the network. We can then get objective and subjective metrics. Can be used different packet loss models and different packetization schemes (number of TS packet per IP packet).

The framework is continuously extended to support other codecs, metrics and probability distribution functions for simulate losses. It is part of a more comprehensive assessment environment that includes other resources like router buffers, flow multiplexing and flow classification. 


\section{ACKNOWLEDGMENTS}

This work has been partially financed by CPUFLIPI Project (MICINN TIN2010-17298), MBACToIP Project, Aragon I+D Agency, Ibercaja Obra Social, Project of Cátedra Telefónica, Univ. of Zaragoza, Banco Santander and Fundación Carolina.

\section{REFERENCES}

[1] Cisco, "Visual Networking Index (VNI)," [Online]. Available: http://www.cisco.com/en/US/solutions/collateral/ns341/ns5 25/ns537/ns705/ns827/VNI_Hyperconnectivity_WP.html. [Accessed 30 mayo 2012].

[2] L. Angrisani y C. Narduzzi, «Testing communication and computer networks: an overview,» IEEE Instrumentation \& Measurement Magazine, vol. 11, pp. 12-24, Oct. 2008.

[3] ITU-T, "Recommendation E.800. Definitions of terms related to quality of services.," Septiembre, 2008.

[4] T. Hoífeld and A. Binzenhöfer, " Analysis of Skype VoIP traffic in UMTS: End-to-end QoS and QoE measurements," Computer Networks: The International Journal of Computer and Telecommunications Networking, vol. 52, no. 3, pp. 650-666, February, 2008.

[5] R. Pries, D. Hock and D. Staehle, "QoE based Bandwidth Management Supporting Real Time Flows in IEEE 802.11 Mesh Networks.," Special Issue PIK on Mobile Ad-hoc Networks, vol. IV/2009, Enero 2010.

[6] M. Fiedler, T. Hossfeld and P. Tran-Gia, "A Generic Quantitative Relationship between Quality of Experience and Quality of Service," IEEE Network Special Issue on Improving QoE for Network Services, 2012.

[7] A. Takahashi, D. Hands and V. Barriac, "Standarization Activities in the ITU for a QoE Assesment of IPTV," IEEE Communications Magazine, February 2008.

[8] S. Winkler, Digital Video Quality - Vision Model and Metrics, John Wiley \& Sons, 2005.

[9] S. Winkler y P. Mohandas, «The Evolution of Video Quality Measurement:From PSNR to Hybrid Metrics.,» IEEE Trans. Broadcasting, vol. 3, n ${ }^{\circ}$ 54, pp. 660-668, Septiembre 2008.

[10] R. Serrat-Gracià, E. Cerqueira, M. Curado, M. Yannuzi, E. Monteiro and X. Masip-Bruin, "An Overview of Quality of Experience Measurement Challenges for Video Application in IP Networks," International Conference on Wired/Wireless Internet Connections, pp. 252-263, Sweden 2010.

[11] Z. Wang, L. Lu and C. Bovik, "Video quality assessment based on structural distortion measurement," Signal Processing: Image Communication, vol. 19, no. 1, January 2004.

[12] M. H. Pinson y S. Wolf, «A new standardized method for objectively measuring video quality,» IEEE Trans. Broadcasting, vol. 50, p. 312, 2004.

[13] C. J. Branden Lambrecht y O. Verscheure, «Perceptual Quality Measure using a Spatio-Temporal Model of the
Human Visual System,» Proc. SPIE, vol. 2668, pp. 450461, March 1996.

[14] ITU-R, "Recommendation BT.500-13.Methodology for the subjective assessment of the quality of television pictures.," International Telecommunication Union, Enero 2012.

[15] J. Greengrass, J. Evans and A. C. Begen, "Not All Packets Are Equal, Part 2: The Impact of Network Packet Loss on Video Quality," Internet Computing, IEEE, vol. 13 , pp. 7482 , March-April 2009.

[16] Y. Lu, Y. Zhao, F. A. Kuipers and P. Van Mieghem, "Measurement Study of Multiparty Video Conferencing," in Proc. of IFIP Networking, Chennai, India, May 2010.

[17] Y. Lu, B. Fallica, F. A. Kuipers, R. E. Kooij y P. Van Mieghem, "Assessing the Quality of Experience of SopCast,» Int. J. Internet Protocol Technology, vol. 4, $\mathrm{n}^{\mathrm{o}} 1$, pp. 11-23, 2009.

[18] R. E. Kooij, F. Nicolai, O. K. Ahmed y K. Brunnström, «Model validation of channel zapping quality,» Proceedings of Human Vision and Electronic Imaging Conference, January 2009.

[19] ITU-T, «Recommendation G.1080. Quality of experience requirements for IPTV services,» Diciembre 2008.

[20] J. Klaue, B. Rathke and A. Wolisz, "EvalVid - A Framework for Video Transmission and Quality Evaluation," 13th International Conference on Modelling Techniques and Tools for Computer Performance Evaluation, pp. pp. 255-272, September 2003.

[21] C.-K. S. W.-S. H. a. A. Z. Chih-Heng Ke, "An Evaluation Framework for More Realistic Simulations of MPEG Video Transmission," Journal of Information Science and Engineering, vol. 24, no. 2, pp. 425-440, March 2008.

[22] A. Lie and J. Klaue, "Evalvid-RA: trace driven simulation of rate adaptive MPEG-4 VBR video," Multimedia Systems, vol. 14, no. 1, pp. 33-50, 2008.

[23] Sirannon, «VQEG STL,» [En línea]. Available: http://vqegstl.ugent.be/?q=node/29. [Último acceso: 10 junio 2012].

[24] A. Rombaut, N. Staelens, N. Vercammen, B. Vermeulen y P. Demeester, «xStreamer: modular multimedia streaming,» de Proceedings of the seventeen ACM international conference on Multimedia, Beijing, China, Octobre 2009.

[25] "FFmpeg," [Online]. Available: http://ffmpeg.org/. [Accessed 1 Junio 2012].

[26] "x264," [Online]. Available: http://www.videolan.org/developers/x264.html. [Accessed 7 Junio 2012].

[27] E. N. Gilbert, "Capacity of a burst-noise channel.," Bell System Technical Journal., vol. 39, Septiembre 1960.

[28] E. O. Elliot, «Estimates of error rates for codes on burstnoise channels.,» Bell System Technical Journal, vol. 42, Septiembre 1963. 
[29] J. McDougall, J. J. John, Y. Yu and L. Miller, "An improved channel model for mobile and ad-hoc network simulations," Communications, Internet and Information Technology, St. Thomas, US Virgin Islands, pp. 352-357, 2004.

[30] O. Hohlfeld, R. Geib and G. Haßlinger, "Packet Loss in Real-Time Services: Markovian Models Generating QoE Impairments," Proc. of 16th International Workshop on Quality of Service (IEEE IWQoS), pp. 261-270, 2008.
[31] "Elephants Dreams," [Online]. Available: http://www.elephantsdream.org/. [Accessed 10 junio 2012]. 